\title{
The moral case for the clinical placebo
}

\author{
Azgad Gold, ${ }^{1}$ Pesach Lichtenberg ${ }^{1,2}$
}

${ }^{1}$ Department of Psychiatry, Herzog Hospital, Jerusalem, Israel

${ }^{2}$ Department of Psychiatry, School of Medicine, Hebrew University of Jerusalem, Jerusalem, Israel

\section{Correspondence to} Dr Pesach Lichtenberg, Department of Psychiatry, Herzog Hospital, POB 3900, Jerusalem 91035, Israel; licht@cc.huji.ac.il

Received 31 December 2012 Revised 7 May 2013 Accepted 22 May 2013 Published Online First 8 June 2013

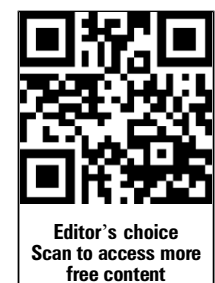

To cite: Gold A, Lichtenberg P. J Med Ethics 2014:40:219-224.

\begin{abstract}
Placebos are arguably the most commonly prescribed drug, across cultures and throughout history. Nevertheless, today many would consider their use in the clinic unethical, since placebo treatment involves deception and the violation of patients' autonomy. We examine the placebo's definition and its clinical efficacy from a biopsychosocial perspective, and argue that the intentional use of the placebo and placebo effect, in certain circumstances and under several conditions, may be morally acceptable. We highlight the role of a virtuebased ethical orientation and its implications for the beneficent use of the placebo. In addition, the definitions of lying and deception are discussed, clarified and applied to the clinical placebo dilemma. Lastly, we suggest that concerns about patient autonomy, when invoked as a further argument against administering placebos, are extended beyond their reasonable and coherent application.
\end{abstract}

\section{INTRODUCTION}

The placebo is arguably the most commonly prescribed drug, across cultures and throughout history. ${ }^{1}$ Yet today placebo treatment has lost its legitimacy, and many might argue that its use in the clinic is unethical, ${ }^{2}$ if not illegal. ${ }^{3}$ Physicians are reluctant to add even potentially effective placebos to their therapeutic arsenal owing to concerns about paternalism, patient autonomy and deception.

We will argue that the intentional use of the placebo, in certain circumstances and under several conditions, can be justified, since it does not involve deception or lying in their common sense. Moreover, we suggest that the moral case for the clinical placebo invites a rethinking of fundamental ethical assumptions about our role as physicians.

\section{THE DEFINITION OF PLACEBO}

The placebo is notoriously difficult to define. ${ }^{4}$ The common reference to 'non-specific' or 'nonbiological' as opposed to specific or biological mechanisms of action remains unrevealing and hints at latent assumptions of a discredited Cartesian dualism, wherein the body has specific mechanisms and the mind is a generic potpourri of mental activity. However, different ways of conditioning for the placebo effect will produce neurophysiological responses which differ by quite specific and defined mechanisms. For example, opioid-conditioned analgesic response to placebo can be blocked by the opioid antagonist naloxone, though naloxone will have no effect on a placebo response which has been conditioned by nonopioid non-steroidal anti-inflammatory drugs. ${ }^{5}$

We prefer to think of the placebo effect from a phenomenological-contextual perspective by way of the biopsychosocial continuum of Engel. ${ }^{6}$ Our focus shifts from a reductionist discussion pertaining to the exact biological or chemical mechanism of the placebo to the phenomenological difference between the clinical settings of prescribing a placebo and a 'regular' medication. We are placing the emphasis upon the physician's intentions at the time of offering treatment, not on the pill itself.

Most medications or procedures are intended to work their effect bottom-up-that is, from a lower to a higher level of complexity. For example, a medication modifies the monoaminergic receptor activity of a neuronal cell. This alters the neurochemical environment of the brain, leading to changes in the emotional subjectivity of the person. When physicians prescribe 'regular' medications, they intend to alter the receptor in order to modify the clinical problem. The patient's subjective condition is addressed by focusing on her parts. The biological dimension in this case is the dominant vehicle of healing.

Placebos, by contrast, are assumed to work top-down. We cannot speak of placebo, or of any treatment for that matter, divorced from its psychosocial context. That context involves the complex background web of cultural norms, semantic and episodic memories, and the interpersonal relationship between treater and treated. Encountering the patient, assuaging anxiety and fostering positive expectations, all percolate down to neurophysiological activity, to receptor function, as brain imaging studies can attest. ${ }^{7} 8$ On a wider, social level as well, roles are played out, expectations are realised, changing fads come into play, the popularity of medications and procedures rises and falls. This too seeps down via the person to having an effect at all levels of the organism. When physicians prescribe placebos they intend to modify the patient's condition by reinforcing the powerful social and psychological healing forces for the benefit of the patient. The biological modification is mediated by psychosocial intervention.

To illustrate the difference between bottom-up and top-down interventions, let us think of a child who injures his hand while playing. The topical antiseptic solution and the bandage are the bottom-up interventions. The boo-boo kiss from his mother is a top-down intervention. Both interventions are real and effective; the first facilitates the recovery of the damaged biological tissue while the second intervention aims at promoting the child's subjective wellbeing and ameliorating his suffering and pain.

A placebo, the common phrase goes, is an inert substance. However, divorced from psychosocial context, subjected to brute physical analysis, the words and sentences on this paper are inert, meaningless shapes too. The neurophysiological substrate which make these entities understandable as writing, the cultural background which makes them 
comprehensible as ideas, the relationship between writer and reader, are what provides these scratches with some semblance of coherence, even meaning.

To phrase it differently, one can speak of a placebo effect without a placebo, as with the hopeful words of the physician. However, one cannot speak of a placebo, certainly not in the clinic, unless it is in the context of a much wider placebo or care effect ${ }^{9}$ or meaning response. ${ }^{10}$ This effect will be an inevitable part of every encounter between physician and patient, which the competent physician will use for the patient's benefit.

At this point, we can redefine the question which we wish to address: when is it ethical, in clinical practice, to offer a therapeutic intervention, when the effect, if any, of that intervention is expected to be mediated by psychophysiological mechanisms, such as expectation, relaxation or conditioned response? ${ }^{11}$

\section{EFFECTIVENESS OF THE PLACEBO}

Physicians, as opposed to non-scientifically oriented therapists, should base their interventions on empirical evidence. Evidence for the effectiveness of placebos derives from brain imaging ${ }^{7} 81213$ and other objective parameters. ${ }^{14-16}$ As effective as placebos sometimes appear to be in research, they may be even more effective in the clinic. The blinding in placebocontrolled studies is often broken, so that subjects know that they are receiving only a placebo. ${ }^{17}$ If so, the negative expectation of receiving a placebo might artificially enlarge the differing responses to medication and placebo.

Similarly, in placebo-controlled trials, the subject is informed that she may receive a placebo. In the clinic, by contrast, the patient presumes that she is receiving a 'real' medication. The uncertainty pertaining to the nature of the medical intervention in research settings may reduce the potential effect of the placebo, as well as of the medication arm of the research. Evidence for this claim derives from the finding that as the number of treatment arms in a specific trial increases, thereby reducing the likelihood of the subject receiving a placebo and increasing his expectation of receiving medication, the effectiveness of both the medication and the placebo increases. ${ }^{18}$ Hence the placebo may be even more effective in the clinic than in the research setting. ${ }^{19}$

The effectiveness of the placebo, where demonstrated, means that a priori prohibiting the placebo deprives patients of an effective mode of treatment, exposes them to side effects of other treatments and incurs extra financial costs.

Nevertheless, it is important to mention that according to an influential 2001 meta-analysis comparing placebo-treatment arms with no treatment, placebos make no clinical difference. ${ }^{20}$ However, the methodological difficulties of combining diverse conditions in a single meta-analysis are serious, and the authors' updated meta-analysis includes more nuanced conclusions. ${ }^{21}$ Furthermore, the meta-analyses implicitly assume the biological reductionist definition of placebo, ignoring the psychosocial context of sickness and health. The subjects in those studies were entered into a research programme and received attention and care. Therefore, in the way that the studies of the meta-analysis were actually conducted, there was plenty of room for the placebo effect without the placebo, which would spuriously suggest that the placebo was no more effective than no treatment, though in fact both had the potential to produce a placebo effect.

\section{FALSE INFORMATION, LYING AND DECEPTION}

Assuming that placebos can be effective, is it ethical to prescribe a placebo, or is the physician guilty of lying and deception? ?2-24 $^{22-2}$
To respond, we would first like to distinguish between providing false information, lying and deception. There are three core components which underlie the discussion pertaining to these concepts: the validity of the provided information, the mindset of the information provider and the mindset of the information recipient.

A lie implies false information, but providing false information is not the same as lying, unless I provide that false information intentionally, not mistakenly. In other words, the negative moral quality of lying requires, first, that the liar believes that what she says is false (the epistemic condition); and second, that the liar provides the false information as if it were true with the intention of making another person believe it to be true (the intentional condition).

Deception can be defined as intentionally causing someone to have a false belief that the deceiver believes to be false' (Carson, ${ }^{22}$ page 46). The deceiver usually has a selfish motive and assumes that causing the recipient to hold a false belief will promote his (the deceiver's) personal interest. As a result, deception is often more complex than lying. In addition, deception differs from lying in two respects: first, as opposed to lying, deception does not necessarily require making a false statement; and second, deception occurs only if the recipient has been successfully convinced by the false belief (Carson, ${ }^{22}$ page 55).

The concept of deception and its relation with lying will be illustrated by the following case: It is $20: 55$; I am rushing with a friend of mine towards the cinema to purchase the last two tickets for tonight's last show. We were earlier surprised to find out that tonight, as an exception, the last show will start at 21:00 instead of the usual 20:00. In front of us in the elevator, we meet a couple planning to see the same movie. We know that if they arrive at the box office before us and purchase the last two tickets, we will miss the movie. But they turn to us and ask if we know when the last showing of the movie begins. My friend replies: 'Usually the last showing starts at 20:00'. They thank us politely and leave the elevator at a different floor. My friend winks at me while trying to conceal his satisfaction.

Was my friend lying? We think not, since he did not provide any false information. The statement 'Usually the last showing starts at 20:00' is true. However, he was certainly deceptive, since he intentionally and successfully caused our competitors for tickets to believe that the movie started at 20:00. Indeed, only by inserting this false belief into the couple's minds were we able to achieve our goal-purchasing the last two tickets.

In deception, the deceiver usually is trying to promote her self-interest by concealing the truth from someone else. On the other hand, many liars are not interested in achieving a welldefined self-serving goal at the expense of another person. For example, if I am asked the time by a stranger and I say 'I don't know' even though I know it is exactly 22:30, I am lying. I intentionally provided false information. However, I do so simply because I am deeply troubled by a personal problem and I do not have the strength to be engaged in any kind of conversation. I am indifferent to whether he believes me or not and I do not have any implicit self-serving purpose behind my lie. In this sense, it seems that some forms of deception are morally worse than lying since the deceiver is more egocentric and selfish.

However, not all forms of deception or lying are selfish or self-serving, as can be illustrated by the following case: My son has a tendency to be late for meetings. I know that he has a very important job interview at 10:00, and he plans to leave the house at 9:00. I know that considering the traffic in the area of the meeting and current weather conditions he should leave an 
hour earlier. However, given his rebellious mood that morning, I assume that he won't accept my advice to leave the house at 8:00. Since his watch is broken, he asks me to tell him when it is 9:00, so he can leave the house. I take advantage of his reliance on my reminder, and tell him at 8:00: 'son, it is 9:00'. He thanks me and leaves the house to the meeting. Based on previous similar occasions, I safely assume that he will be grateful to me in hindsight.

In this case, I was both lying and deceiving. I intentionally provided false information that I knew was false. It was crucial that my son believe that it was indeed 8:00. At the same time, the moral quality of my action, we argue, is not as bad as 'classic' lying or deception. In fact, given the fact that my sole intention was my son's benefit, the core component of selfishness that usually characterises deception is absent.

But what if I tell my son at 8:00: 'I believe it is time for you to leave', instead of the explicit lie 'it is 9:00'? In this case, I am not lying since I do not intentionally provide false information. Nevertheless, it is fair to argue that I am not being entirely honest with my son, since I know that he is most likely to interpret my statement as equivalent to 'it is 9:00'. That being said, the falsehood of the content of my statement depends on my son's potential interpretation. There is nothing inherent in my statement that is false. Moreover, I wholeheartedly stand behind the content of my statement since I do believe that my son should leave the house at the time I told him to. In addition, my statement is sufficiently different from deception, in two ways: first, my primary intention is not making my son believe it is 9:00, although it may become a by-product of my statement; second, I am motivated by achieving my son's goal (finding a job) and not mine. I decided that my foremost duty towards my son is not providing him with accurate information but rather helping him accomplish his ultimate goal. I do not relish misleading my son, but I am willing to sacrifice my moral comfort in order to promote his welfare. From a moral perspective, whether this action can be defined as deceptive or not, we think that dishonesty of this sort is substantially different from the standard proscribed sort of deception.

\section{THE ETHICS OF PLACEBO TREATMENT}

Is the physician lying to, or deceiving, the patient by providing a placebo instead of 'real' medication?

If the physician knows that this is a placebo, yet she intentionally says to the patient that this is not a placebo but a medication, she is lying. In addition, if the patient believes her physician that the placebo is a 'true' medication, then there is also a deception involved in this situation. This form of placebo treatment should be prohibited for both tactical and moral reasons.

A much more acceptable form of placebo treatment is when the placebo is offered in a way that allows full disclosure. The most obvious way to do so is to state that you, the patient, are now receiving a placebo. Some research has suggested that full disclosure, free of any trace of lying or deception, can be provided without impugning the effect of the placebo. ${ }^{25}$ The provided information is true and there is no attempt by the physician to insert into the patient's mind false beliefs. Though some elements of suggestion and persuasion probably contribute to the positive outcomes, it seems that this form of placebo treatment, especially for clinical conditions where the best treatment choice is uncertain, is appropriate.

Besides these two relatively simple cases, there are other more complicated forms of placebo treatments that should be dealt with. For example, the physician might say, 'I am prescribing a pill which research suggests can be of benefit to you. In your circumstances I have reason to believe that it will work, with a minimum of side effects'.

The hesitation some feel with this approach is based on the assumption that we are deceiving the patient because she thinks she is getting 'real' treatment, but she is not; she is getting a placebo. This view seems to be supported by the American Medical Association, according to which physicians are prohibited from providing 'a substance ... that the physician believes has no specific pharmacological effect (our emphasis) upon the condition being treated'. ${ }^{26}$ This is once again the Cartesian fallacy of a mind-body dualism entering through the back door. If one realises that the placebo effect is-in those cases where evidence has accrued for its effectiveness-as real as responses to pharmacology, placebo treatment may gain therapeutic legitimacy. The criteria for evaluating the moral status of an intervention should focus on the question whether the physician believes-based on empirical data-the intervention will be effective; the effectiveness of an intervention should not be limited to its 'specific pharmacological effect'. The everyday medical practice is abundant in situations in which physicians provide interventions whose specific pharmacological effect is unknown.

In light of our definitions of lying and deception, the legitimacy of several forms of placebo treatment depends in part on the mindset of the physician. If the physician truly believes in the therapeutic effect of the placebo then she holds her statement above to be true. As a result, this form of placebo treatment cannot be considered lying. In addition, if we define deception as intentionally causing someone to have a false belief that the deceiver believes to be false', then for the physician who genuinely believes in the therapeutic qualities of the placebo treatment, it would not be considered deception as well. Nevertheless, since the patient may interpret these vague statements as if a 'true' medication has been offered, some physicians may still feel dishonest about adopting this strategy. We understand this concern; however, following our argument in the father and son example, this form of dishonesty may be accepted in cases in which alternative strategies are expected to prevent the patient from achieving her ultimate goal-to feel better. $^{3}$ Indeed, this act may be regarded as paternalistic. We will deal with this challenge below. For now, we will note that some attenuated paternalism should not be automatically deemed as unethical. Despite its bad reputation in the past few decades in medical ethics, pater-nalism, as the name implies, can sometimes represent the mode of action which a compassionate father would choose in order to promote his child's welfare. When these actions are being undertaken by physicians out of a genuine benevolence, patients may appreciate their motives as positive.

Thus far, we have argued that under the situations and conditions described above, placebo treatment does not involve deception or lying, and therefore may be ethically justified. It is worth emphasising that our argument differs from previously presented justification for the use of clinical placebo ${ }^{19}$ in the sense that in our view, the use of placebo under the abovementioned conditions should not be considered deceptive. Hence, the term 'deceptive placebo' which is often used in this context is not accurate. However, even if one were to accept that placebo is deceptive, its use might be theoretically justified. In the classic form of deception, the deceiver derives self-serving benefit by deceiving. A physician offering a placebo in order to be free of an irksome patient would be guilty of deception in that immoral sense. However, a physician who in a non-selfish 
manner believes that by providing a placebo, she is helping bring relief to her suffering patient, could arguably defend her action as a fulfilment of her duty as a physician. The non-selfish nature of this kind of deception makes it significantly and qualitatively different from the classic morally faulty form of deception, at least from a virtue-based perspective.

The objection to beneficent or altruistic deception will be that by unilaterally deciding the good of the patient while denying him full disclosure the physician acts paternally and violates the patient's autonomy. We will respond to this claim.

\section{CLINICAL PLACEBO, PATIENT AUTONOMY AND PATERNALISM}

Patient autonomy has become a central tenet of medical ethics in the past 60 years. Following the horrendous abuses of human rights conducted by German Nazi medical doctors experimenting on concentration camp inmates, the Nuremberg Code in 1947 established new standards in medical ethics to prevent such abuses. After the 1972 revelation of the Tuskegee Syphilis Project, in which male Afro-Americans diagnosed with syphilis were left untreated in order to follow the natural progression of the disease, new standards of medical ethics were promulgated, requiring that research subjects be treated as autonomous subjects.

The principle of autonomy has been transferred to the area of clinical practice as well. The standards of autonomy have greatly advanced the ethical practice of medicine, and prevented unimaginable abuse. Nevertheless, today, placebo is considered by many to be beyond the pale of clinical legitimacy, since it violates patient autonomy. Is this approach justified?

Clearly certain essential components of medical interventions must be disclosed to all patients, even if full disclosure is likely to lead the patient to make what from the physician's perspective is the 'wrong' decision. These components include, among others, significant side effects of proposed interventions. The informed consent legal doctrine was developed largely in response to cases in which this sort of disclosure was missing. ${ }^{3}$ When a physician even with good intentions withholds this sort of information, which is meaningful to most patients, he is being paternalistic in the wrong sense.

However, debate remains as to what constitutes essential information that physicians must disclose to patients. ${ }^{27}$ This derives from uncertainty about what most patients are interested in hearing from their physicians. ${ }^{3}$ The case of the placebo is especially challenging, since the withheld information refers not to physical side effects but rather to the placebo's mechanism of action (according to our formulation, top-down rather than bottom-up). In addition, it is unclear whether the physician needs to provide information that would satisfy the 'reasonable' patient or whether a more subjective threshold should guide physicians-namely, what would this specific patient want to hear before undergoing a medical intervention. For example, the country in which a medication has been manufactured seems to be considered peripheral information that physicians are not required to disclose to their patients (in fact, most physicians do not know). However, for the individual patient this information may be extremely important and essential; for example, a Holocaust survivor, who avoids purchasing any kind of goods from Germany, might be furious if his physician has not disclosed that the medicine he has been prescribing for years was manufactured in Germany.

We think that when a physician is aware of such idiosyncratic considerations she is not allowed to intentionally conceal this kind of information, usually considered peripheral, even if it is concealed with the most benevolent of intentions. The same argument applies to placebo or placebo-like interventions. We do not even know that most patients prefer to always have full disclosure. The demand that autonomy remain uncompromised wherever possible is itself a violation of autonomy, by forcing upon the patients a value they may not necessarily share. Whether most people hold autonomy to be a central value, and would indeed prefer to be informed about the mechanism of action of interventions even when no extra physical risk is involved and when this disclosure may compromise the effectiveness of the intervention, remains an empirical question largely uninvestigated. Therefore, unless the physician knows, or reasonably assumes, based on personal acquaintance, that her patient is interested in being informed about the mechanism of action of the proposed intervention, she is not obligated to disclose this sort of information. When the preferences of the individual patient for the extent of disclosure are unknown, disclosing potentially unneeded information may be as paternalistic as concealing such information.

This is so when the physician genuinely believes in the therapeutic effect of placebos and is motivated by benevolent intentions. If there is no certainty or likelihood that the patient is going to feel upset about not having been informed that he is receiving a placebo, the paternalistic quality of this nondisclosure is akin to not providing the information pertaining to the country in which the medication has been manufactured. In both cases, the physician may be justified in assuming that this sort of information is peripheral and of minor significance to the patient.

More broadly, far beyond the clinical placebo dilemma, the encounter between physician and patient is inherently fraught with withheld knowledge. For example, skilled physicians use various techniques to establish a rapport with their patients. They may allow their patients to speak freely in order to gain their trust, without explicitly saying: 'you should be informed that I do not interrupt you so you'll think that I am really interested in your problem'. That truthful but absurd statement would compromise the ultimate beneficent therapeutic goal and therefore we find it undesirable.

Let us consider another example. The placebo effect accounts for $30-100 \%$ of the effectiveness of antidepressant drugs. ${ }^{28}$ Yet are doctors expected to tell this to their depressed patients who seek relief for their suffering? Are they considered deceitful for not sharing this information? Even if the patient asks how the medication works, the doctor will probably talk about brains and serotonin. We do not see any deception or undesirable paternalism in that. We do not know that patients would like to receive this sort of information, which will make them more knowledgeable but will risk the effectiveness of the treatment. Some patient may find this information essential, but others may regard it as peripheral and even undesirable. Patients come to physicians first and foremost to feel better, not to become more knowledgeable or autonomous in the legal sense. ${ }^{3}$

Scholars who argue that placebo or placebo-like effects should be disclosed to patients ${ }^{29}$ probably assume that patients are interested in obtaining as much information as possible for all aspects of the medical intervention they are about to undergo, or alternatively, they consider information about placebo mechanisms of action essential. Their argument is more persuasive in cases in which a relatively more risky intervention is at stake, such as electroconvulsive therapy. ${ }^{30}$ It is less convincing when there are no apparent physical consequences involved, such as in revealing techniques of communication style, especially when the full disclosure may sabotage the 
effectiveness of the treatment. For patients who have made explicit their wishes to be fully informed about placebo effects, or for others who the physician has reason to believe would be interested in such information, concealing the placebo would be an immoral violation of patient autonomy. But for most patients, the placebo is merely one example among many where full disclosure is not, and need not be, provided.

\section{BACK TO VIRTUE-BASED ETHICS}

How did autonomy, in its expansive version, achieve this virtually unchallenged hegemony in the field of medical ethics? As the central arbiter of medical ethics, autonomy has assumed tyrannical dimensions which set an unattainable standard while deeply distorting the way in which medicine is practised. ${ }^{31}$ In a liberal society, different values will inevitably conflict, and ethics strives to provide a method for resolving these conflicts. ${ }^{32}$ In medicine, the reflexive moral priority of autonomy over beneficence has been exaggerated.

Where have we gone wrong? Throughout most of history, virtue ethics has been a prominent pillar of ethical thinking. ${ }^{33}$ This goes back to the time of the ancient Greeks, for whom ethics was a question of whom the person is. The ability to navigate through conflicting demands was called phronesis, which is the practical wisdom necessary to translate virtue into the right action.

The aftermath of the Holocaust and Tuskegee Syphilis Project, as representative events, yielded a survivors' guilt which was translated into a tremendous effort to restore the main victim: human autonomy. The virtuous cultivation of the physician was no longer the central point of medical ethics. The result has been a proliferation of guidelines, rules and codes, instructing us how to act. The doctrine of informed consent, while certainly contributing to the ethical practice of medicine, rose to dominance at the expense of other professional values. Doctors have traditionally chosen their field of activity with a sense that they were blessed with the opportunity to be beneficent, to ameliorate suffering. At its best, medicine could be a calling, even one suffused with a moral sanctity. ${ }^{34}$ While the gains in ethics borne of respect for patient autonomy are indubitable, the pendulum may have swung too far in that direction, where rules are paramount and virtue is at best quaint. We suspect that the $15-80 \%$ of physicians who use placebo treatments ${ }^{35} 36$ are at least occasionally acting out of the same sense of moral imperative, their learned phronesis, which enables them to balance the conflicts of autonomy with beneficence.

One might object that this approach permits too much scope for personal latitude among physicians. One might also wonder whether physicians are capable of discerning patients' views on these matters. These are reasonable concerns that should be dealt with both empirically (eg, finding valid methods to accurately estimate patients' preferences based on their overt behaviour or psychosocial characteristics) and educationally (teaching physicians what is considered an appropriate application of professional values). We expect that once physicians are enabled to conduct their practice in the way we have described here, a new phronesis that balances the values of beneficence and autonomy will gradually and naturally arise.

\section{CLINICAL PLACEBO: GENERAL GUIDELINES}

An integral part of the phronesis involved in placebo treatment relates to the art of maintaining patient trust in the physician. This trust is one of the most important forces that make the placebo effect possible. From a utilitarian perspective, it would be a dreadful mistake to risk the patient's trust in his physician for the possibility of achieving a placebo effect by a placebo treatment.

Therefore, it seems crucial to establish a sustainable rapport with patients and to have a good sense of their expected reaction before offering placebo treatment.

Despite our fundamental differences with the direction of medical ethics, the ramifications for practical recommendations may not be great. Balancing the claims of autonomy, beneficence, respect, justice and honesty, the following rough guidelines concerning the use of placebos can be offered:

- The intentions of the physician must be benevolent, and her only concern the well-being of the patient.

- The placebo cannot be given in place of another medication that the physician reasonably expects to be more effective.

- Administration of placebos should be considered when a patient is refractory to standard treatment, suffers from its side effects, or is in a situation where standard treatment does not exist.

- The placebo can be named for what it is, but this is not required. It is then sufficient to make a statement along the lines that the substance provided has been found to be effective for the problem at hand, even though the mechanism is uncertain.

- Placebos should only be used where research has provided empirical evidence to expect them to have the potential of providing relief (eg, for pain, depression, etc).

- When proved ineffective the placebo should be withdrawn.

- The physician should not lie. She should respond honestly when asked about the nature and expected effects of the placebo treatment she is offering. In addition, an explanation of top-down interventions is appropriate and may serve as an educational corrective to dominant chemo-centric tendencies in medicine.

\section{SUMMARY}

Placebo treatment may be ethically permissible under certain situations and conditions. In some cases, especially when the physician believes in the therapeutic qualities of the placebo, the motivation for the treatment stems from a genuine concern for the patient's well-being and the treatment is not presented to the patient in false manner, placebo treatment should not be considered deceptive.

In a broader sense, the complex interplay of conflicting values, and the beneficence which remains the inspiration for so many to become a doctor, will better be served by a virtue ethics which can permit the sparing use of placebos in certain circumstances.

Acknowledgements We thank Dr Pekka Louhiala and the two anonymous reviewers for their insightful comments to the first draft of this manuscript.

Contributors PL: guarantor of the paper, provided the original impetus for writing the paper and contributed mainly to the definitions of placebo and the analysis of placebo effectiveness. AG: contributed to the ethical analysis and placed the discussion in the context of classical conceptions of ethics.

Competing interests None.

Provenance and peer review Not commissioned; externally peer reviewed.

\section{REFERENCES}

1 Shapiro AK, Shapiro E. The powerful placebo: from ancient priest to modern physician. Baltimore: Johns Hopkins University Press, 1997.

2 Louhiala P. The ethics of the placebo in clinical practice revisited. I Med Ethics 2009;35(7):407-9.

3 Kolber AJ. A limited defense of clinical placebo deception. Yale Law Policy Rev 2007;26(1):75-134

4 Gøtzsche PC. Is there logic in the placebo? Lancet 1994;344(8927):925-6. 
5 Amanzio M, Benedetti F. Neuropharmacological dissection of placebo analgesia: expectation activated opioid systems versus conditioning activated specific subsystems. J Neurosci 1999;19:484-94.

6 Engel GL. The need for a new medical model: a challenge for biomedicine. Science 1977:196:129-36.

7 Mayberg HS, Silva JA, Brannan SK, et al. The functional neuroanatomy of the placebo effect. Am J Psychiatry 2002;159(5):728-37.

8 Petrovic $\mathrm{P}$, Kalso E, Petersson KM, et al. Placebo and opioid analgesia-imaging a shared neuronal network. Science 2002;1;295(5560):1737-40.

9 Louhiala P, Puustinen R. Rethinking the placebo effect. Med Humanit 2008;34:107-9.

10 Moerman DE, Jonas WB. Deconstructing the placebo effect and finding the meaning response. Ann Intern Med 2002;136:471-6.

11 Lichtenberg $\mathrm{P}$, Heresco-Levi U, Nitzan U. The ethics of the placebo in clinical practice. J Med Ethics 2004;30:551-4.

12 de la Fuente-Fernández R, Ruth TJ, Sossi V, et al. Expectation and dopamine release: mechanism of the placebo effect in Parkinson's disease. Science 2001;293:1164-6.

13 Bingel U, Lorenz J, Schoell E, et al. Mechanisms of placebo analgesia: rACC recruitment of a subcortical antinociceptive network. Pain 2006;120(1-2):8-15.

14 Benedetti F, Amanzio M, Baldi S, et al. Inducing placebo respiratory depressant responses in humans via opioid receptors. Eur J Neurosci 1999;11(2):625-31.

15 Reeves RR, Ladner ME, Hart RH, et al. Nocebo effects with antidepressant clinica drug trial placebos. Gen Hosp Psychiatry 2007;29(3):275-7.

16 Pressman A, Avins AL, Neuhaus J, et al. Adherence to placebo and mortality in the Beta Blocker Evaluation of Survival Trial (BEST). Contemp Clin Trials 2012;33 (3):492-8.

17 Boutron I, Estellat C, Guittet L, et al. Methods of blinding in reports of randomized controlled trials assessing pharmacologic treatments: a systematic review. PLoS Med 2006:3(10):e425.

18 Sinyor $M$, Levitt $A J$, Cheung $A H$, et al. Does inclusion of a placebo arm influence response to active antidepressant treatment in randomized controlled trials? Results from pooled and meta-analyses. J Clin Psychiatry 2010;71(3):270-9.

19 Foddy B. A duty to deceive: placebos in clinical practice. Am J Bioeth 2009:9(12):4-12.
20 Hrobjartsson A, Gotzsche PC. Is the placebo powerless? An analysis of clinical trials comparing placebo with no treatment. N Engl J Med 2001;344:1594-602.

21 Hróbjartsson A, Gøtzsche PC. Placebo interventions for all clinical conditions. Cochrane Database Syst Rev 2010;(1):CD003974.

22 Carson TL. Lying and deception. New York: Oxford University Press, 2010.

23 Martin C. The philosophy of seception. New York: Oxford University Press, 2009.

24 Bok S. Lying: moral choice in public and private live. New York: Pantheon Books, 1978.

25 Kaptchuk TJ, Friedlander E, Kelley JM, et al. Placebos without deception: a randomized controlled trial in irritable bowel syndrome. PLOS ONE 2010;5(12):e15591.

26 Bostick N, A Sade R, Levine MA, et al. Placebo use in clinical practice: Report of the American Medical Association Council on Ethics and Judicial Affairs. J Clin Ethics 2008;19(1):59-61.

27 Barnhill A. What it takes to defend deceptive placebo use. Kennedy Inst Ethics 2011;21(3):219-50

28 Khan A, Faucett J, Lichtenberg $P$, et al. A systematic review of comparative efficacy of treatments and controls for depression. PLoS One. 2012;7(7):e41778.

29 Blease $C$. The principle of parity: the 'placebo effect' and physician communication. J Med Ethics 2012:38(4):199-203.

30 Blease C. Electroconvulsive therapy, the placebo effect and informed consent. J Med Ethics 2013:39(3):166-70.

31 Gold A. Physicians' "Right of Conscience"-Beyond Politics. J Law Med Ethics 2010:38(1):134-42.

32 Berlin I. The pursuit of the ideal. In: Hardy H, Hausheer R, eds. The proper study of mankind: an anthology of essays. Pimlico, 1998:1-16.

33 Hursthouse R. "Virtue Ethics", The stanford encyclopedia of philosophy (Summer 2012 edition). In: Zalta EN. ed., http://plato.stanford.edu/archives/sum2012/entries/ ethics-virtue/

34 Marcum J. The virtuous physician: the role of virtue in medicine. Dordrecht, New York: Springer, 2012

35 Nitzan U, Lichtenberg P. Questionnaire survey on use of placebo. BMJ 2004;329 (7472):944-6.

36 Fässler M, Meissner K, Schneider A, et al. Frequency and circumstances of placebo use in clinical practice-a systematic review of empirical studies. BMC Med 2010;23(8):15. 